\title{
PEACE SUPPORT OPERATIONS IN SUB- SAHARAN AFRICA: LIVED EXPERIENCES OF EMERGENCY CARE PROVIDERS DURING EXTERNAL DEPLOYMENT
}

\author{
Tshikani Lewis Khoza, ${ }^{1}$ Nombeko Mshunqane ${ }^{2}$ \\ and Simpiwe Sobuwa ${ }^{3}$ \\ Durban University of Technology
}

\begin{abstract}
Military emergency medical care in support of mission personnel in operational areas serves as a vital element of peace support operations, as it ensures the maintenance of the human resources deployed in mission areas. Although the South African National Defence Force is facing significant financial constraints, it is becoming increasingly mobile, as South Africa continues to pledge military and diplomatic assistance in the African continent to maintain peace and stability, promote development and provide security. Consequently, when deployed abroad, military emergency care providers face unique challenges.
\end{abstract}

The aim of this paper is to describe the lived experiences of Gauteng-based emergency care providers in delivering emergency medical care amid resource constraints during external deployment. The descriptions were extracted from a larger study titled "exploring lived experiences of Gauteng-based military pre-hospital emergency care providers during external deployment". The research was conducted using a qualitative, exploratory research design and was guided by a phenomenological approach.

The findings indicate that Gauteng-based military emergency care providers often did not have enough equipment or appropriate transportation to perform their duties during external deployment. As a result, they are hindered from providing quality care to ill and injured patients.

Key words: Military experiences, external deployment, emergency medical care

\section{Introduction}

Peace support operations are categorised as peace building, peace enforcement and peace-making, peacekeeping or, collectively, peace support. ${ }^{306}$ Peace support operations are conducted in support of diplomatic efforts to restore and maintain peace in conflict areas often under unfavourable political and economic conditions to accomplish political objectives determined on a diplomatic level and to provide a means of achieving a political solution to problems through the use of military 
force. ${ }^{307,308}$ Operations are carried out within the guidelines and boundaries set by the United Nations (UN), the African Union (AU), guerrilla forces and other parties once consent has been granted by the host country to deploy peacekeepers in their territory. These guidelines are important in respecting and promoting the sovereignty of UN member states. ${ }^{309}$

Healthcare services provision in support of mission personnel in operational areas forms an integral part of peace support operations. These services ensure the physical and mental wellbeing of deployed personnel, the maintenance of human resources and the preservation of life, whilst also reducing the morbidity and mortality of the soldiers deployed in mission areas. ${ }^{310}$ Accordingly, the duties entrusted to military emergency care providers include rendering emergency medical care (EMC) and primary healthcare, as well as evacuating casualties resulting from peace support operations. ${ }^{311}$

A study by Shilcutt highlights that the provision of healthcare services in support of mission personnel in operational areas also offers a strategic military advantage that military commanders can use to win battles in that, by saving the lives of the severely injured and treating those with minor injuries, they may continue fighting. ${ }^{312}$ As the fourth arm of service of the South Africa National Defence Force (SANDF), the core aim of the South African Military Health Service is to provide multidisciplinary health care, which includes EMC, to members of the SANDF and their dependents during peacetime, wartime, military operations, disaster relief and humanitarian operations. $^{313,314}$

Although countries such as Central African Republic (CAR) have been in a state of civil war and instability even before the cold war and still continue to this day, the post-cold war era in Africa has been engulfed with ethnic cleansing, revolt and rebellion against dictatorships and one-party states previously installed by the colonial governments. This led to civil war in many African states such as Democratic Republic of Congo (DRC), Liberia, Angola, Burundi, Sudan, Nigeria, Chad and Somalia. ${ }^{10}$ While some countries went into full-scale civil war, in others guerrilla leaders became warlords and continued the plunder of the natural resources whilst exploiting the masses. This fuelled South Africa's entry into peace support operations in Africa under the auspices of the UN. As international communities such as the United States of America and France continue to decrease their military presence in Africa, so South Africa has pledged military and diplomatic assistance in maintaining peace and stability, promoting development and providing security in the continent. ${ }^{315,316}$ This has led to the SANDF increasing its presence in remote geographical areas in an effort to meet their mandate, all the while facing significant financial constraints. ${ }^{317,318}$ Financial constraints, which result in resource constraints, present military emergency care providers with unique challenges when deployed externally and, hence, warrant a unique approach to the provision of prehospital emergency care ${ }^{319}$ Additionally, the provision of EMC amid resource constraints is a lived experience of externally deployed military emergency care providers. ${ }^{320}$ 
The aim of this paper is to discuss the descriptions of the lived experiences of Gauteng-based emergency care providers providing EMC amid resource constraints during external deployment. The descriptions were extracted from a larger study titled "exploring the lived experiences of Gauteng-based military pre-hospital emergency care providers during external deployment". The study aimed to explore and describe these lived experiences of Gauteng-based military prehospital emergency care providers (PECPs) when providing EMC when deployed abroad.

\section{Method}

The study was conducted using a qualitative, exploratory design guided by social phenomenology. According to Creswell, a phenomenological approach is used to describe what a population experiences and how they interpret their experiences of a particular phenomenon. ${ }^{321}$ According to Polit and Beck, exploratory designs illuminate the way in which a phenomenon manifest. ${ }^{322}$

The study was conducted within four South African Military Health Units based in Gauteng Province, namely, 7 Medical Battalion Group, Area Military Health Unit Gauteng, 8 Medical Battalion Group and the School for Military Health Training, during August 2016. The data was obtained from semi-structured, in-depth individual interviews held with eighteen $(n=18)$ PECPs enlisted in the South African Military Health Service based in Gauteng. Written informed consent was obtained prior to the interviews being conducted. Purposive, random sampling was used to obtain 25 participants from the 276 PECPs who provide military EMC in Gauteng. The purposive sampling method employed a type of non-probability sampling where the decisions in relation to the individuals to be included in a study are taken by the researcher. ${ }^{323}$ The researchers used eligibility requirements as contained in the inclusion criteria and assessed them by using the participant sign-up form, as advised in a paper by Morgan and Given, to purposefully select 55 participants who had been deployed externally from the four units. ${ }^{324}$ Accordingly, a sample of 25 participants was randomly selected using the Microsoft Excel randomisation tool. As a result of data saturation, data collection was terminated after 18 participants had been interviewed.

Participants had to be full-time members of the SANDF with a minimum of two years' experience and should have been externally deployed. These participants were chosen as they could provide a comprehensive account of their experiences as PECPs during external deployment. The data collection tool was pre-tested prior to data collection with three PECPs working at the Area Military Health Unit in KwaZuluNatal. The pre-testing gave an opportunity to refine the interview skills and to ensure that the interview questions were appropriate.

Ethical clearance to conduct the study was provided by the higher education institution through which the study was undertaken (IREC 45/16). Gatekeeper permission was granted by the 1 Military Research Ethics Committee (IMH/302/6/01.02.2016) as well as Defence Intelligence (DI/SSDCI/DDS/R/202/3/7). 
Thematic analysis was used to analyse the data. This involved searching for themes that emerged which were key to describing the experiences of PECPs working at the South African Military Health Service, Gauteng. The process of thematic analysis started when themes were noted during data collection, continued while data was transcribed and ended when the themes were reported. Data was transcribed verbatim and the transcripts uploaded onto NVivo Computer Aided Qualitative Data Analysis Software. The researchers familiarised themselves with the data by reading the transcriptions repeatedly whilst listening to the original audio interviews to check for accuracy. The researchers also noted down and brainstormed their initial ideas, which allowed them to immerse themselves in the data. ${ }^{325,326,327}$

Initial codes, some of which were established during data collection, were produced from the data and organised into nodes in NVivo. Further codes emerged during the data analysis as the researchers immersed themselves in the data. When all the data had been coded, the codes identified were combined in NVivo to form broader themes -some forming the main themes and some the sub-themes, and some were categorised still further within the sub-themes.

\section{Results}

With regard to experience, the study identified that 12 participants had between six to ten years, two had between 20 and 25 years and another two had between 11 and 15 years. The remaining two participants had between three- and five-years' experience.

Five themes emerged from the interviews that were conducted. However, this paper discusses only the results related to the themes "Resources" and "Political situation during external deployment" and the subthemes within these themes. "Resources" here refers to the tools and equipment that military emergency care providers require to perform their duties during external deployment. However, the theme "Political situation during external deployment" refers to the political state of affairs in the host country during the external deployment period. The two subthemes that emerged with regard to the theme "Resources" were "limited resources" and "unique casualty transportation". The four subthemes that emerged regarding the theme "Political situation during external deployment" were "political will of the host country", "civilian hostility", "gaps between what the mandate authorises" and "prolonged casualty evacuation times". Although in the main study, five subthemes emerged within the theme "Political will of the host country", this paper omits the subtheme "gaps between what the mandate authorises" as it provides no pertinent information to achieving the aim of this paper.

\section{Resources}

\section{Limited resources}

The majority of participants indicated that they had been poorly resourced during external deployment and that they often lacked the essential lifesaving equipment that 
they required to do their jobs, and thus they had to improvise when providing their patients with EMC. One participant described their experiences as follows:

I encountered a lot of challenges because we were using the ambulance that side that didn't have equipment. The only equipment I had was my medical bag, with the medication [which] I stocked mo [Sesotho: here], here in South Africa before I left [Participant 10].

Another combat medic highlighted that the equipment that was available was outdated and some of it was damaged:

So, you go to external deployment your, you, I'm going to find the same equipment that I saw 2013 or even before is still in there. It's broken, we are fixing. So, I understand they say medical equipment is expensive but it's a must have ... so, if we can get equipment then everything, I think, will be fine. Medical bags, you go there, I'll find the same bag I was using 2011, not even 2013, I'm still going to find the same medical bag [Participant 1].

There were also challenges involved in receiving equipment that had been ordered. It was also found that PECPs often had to purchase supplies such as medication using their own funds in order to ensure EMC provision. One participant stated:

There was a point at which we had to buy from our own pockets as emergency medical care providers, including the doctor, we had to pop out some money, buy vacoliters and admin sets because we didn't have but, otherwise, we just, literally, medication and equipment, because when we do our inspection you find that we, we are supposed to have certain things at the sickbay but then we do not have and it's not promising [certain] that we, we can get those things within the period that we are deployed for [Participant 14].

The participant also highlighted the practice of borrowing equipment from other bases during inspections to mislead inspectors regarding the current state of preparedness:

The minute you get there, I just felt like they forget about you, like I said previously about the equipment and, every time when we have inspection, we need to borrow from a certain base just to make as if we have everything, but we didn't really have everything because, after that inspection you return that. So, what are you left with, basically, you're not combat ready [Participant 14].

\section{Unique casualty transportation}

This study revealed that participants had to use combat vehicles to transport patients owing to the shortage of ambulances. One participant reported that: 
My second one was in Sudan where we didn't have ambulances at all, we had to use mambas [armoured personnel carrier]; caspirs [armoured ambulance] were there as ambulances but they were unserviceable... So, we had to use mambas which are a transport that transports troops to the bush for patrols... it's not an ambulance. If I had an emergency, it was much of a hassle [Participant 10].

While another participant reported:

Even the vehicles, sometimes the vehicles, you find one vehicle there, other vehicles are broken and then you must utilise other means of transport when you are there, not even your specific marked medical vehicle, you must use the combatants'vehicle to move around [Participant 3].

The combat vehicles are not suitable for patient care and are not marked with either the Red Cross or the Red Crescent and, hence, do not comply with the requirements of the Geneva Convention for the transportation of patients.

\section{Political situation during external deployment Political will of the host country}

Participants highlighted that a lack of political will of the host country often causes delays in the provision of supplies and a lack of resources. They reported that logistical flights were not given landing rights, which delayed the arrival of supplies and resources. One participant reported that:

If you want to get medicine into a certain country you must wait for, for that peoples' foreign affairs members to allow, sometimes, an aeroplane into the, the country. That's why, sometimes, there are, sometimes, there are challenges because, sometimes, you get medic, uh, the support, uh, the supporting things like medicine or equipment, sometimes you get it little bit late, but that doesn't mean SAMHS doesn't support you. The thing [cargo aircraft] is on the ground, it stands at the airport, but it can 't, for example; maybe land in that country because of the, uh, landing rights of certain countries [Participant 16].

Another participant reported that:

Whenever we need to get, eh, our, our parcels from South Africa here you find that they tell you that there are no flights that are allowed to land that side, so, sometimes, also with the, with the support maybe, eh, eh, the things like the logistical things that we needed, it was also a challenge, like, to get because, like, eh, the Sudanese, the way they operate is totally different to the way we operate ... [Participant 18].

One participant also highlighted that, at times host countries used religious observances as a justification for providing low political support to the mission: 
The only thing that we were having challenges about it was, eh, medication, shortage of medication but we managed to deal with the medication that we had, so we were using what we have, like, for instance, let me just make an example with ops medics, uhm, you find that you have, you don't have enough, eh, equipment or you don't have equipment, you'll improvise, so whatever that we were having that side, we used it because we were told that we have challenge with the plane to go from here to Sudan. Those eh, eh, eh, things that the Sudanese are doing, like, uh, there mustn't be any plane in because of these Ramadan things so... [Participant 2].

\section{Civilian hostility}

Participants reported that their lack of resources within the mission areas led to them taking the initiative to equip themselves and consequently exposing themselves to financial exploitation by the local population. One participant reported that:

I mean, it was terrible, even if you have to buy the medication you must go in there and then you are not allowed and you must go in and check what you need and then some way, somehow, because we are foreigners they were giving us less, eh, we were buying medication - very high, highly expensive, ja[Participant 15].

\section{Prolonged casualty evacuation times}

The findings reveal that PECPs often cared for patients for prolonged periods in the pre-hospital setting without essential lifesaving resources. One participant described their experience as follows:

...sometimes you get stuck and then you have to stay overnight away from the base. Eh, sometimes you get ambushed, for instance, nhe, and then still you can't go back to the base and then some of the patients need medication right there and then and then, when it comes to emergency drugs, unfortunately nothing [Participant 9].

...transportation, it's a challenge, most of the times with own forces it's very far from the base because we drive long distances to do your laers [Afrikaans: laager], like to transport trucks and all that, so the challenge was the resources and transportation, mostly [Participant 17].

Combat medics also experienced prolonged waiting times to obtain authority to evacuate casualties by air. One participant reported that:

when I got injured..., I waited for two weeks for the chopper to come and take me to the level two hospital [Participant 10].

Due to prolonged waiting times to obtain authority for air evacuation, military commanders often resorted to road transportation to get the job done: 
It will take you two days to transport the patient to, to a hospital, you see, because the terrain and also the transport, because you cannot transport the patient from where we were situated with the mamba, for a distance of about 300 kilos [kilometres], you see, you will drive for five days. It was a challenge [Participant 18].

\section{Discussion}

The lived experiences of the resource constraints reported by the PECPs responsible for providing EMC during external deployment give an indication of the challenges emergency care providers may encounter when deployed during peace support operations in the sub-Saharan African region. Under these conditions, such resource constraints are an obstacle to the effective provision of EMC to ill and injured patients. In addition, this adversity is further exacerbated when host countries offer inadequate political support to the mission.

The participants in this study reported that they often have insufficient equipment to perform their duties. Sigri and Basar assert that operational resources such as healthcare equipment are often scarce during external deployment. ${ }^{328}$ Similarly, studies by Chapman et al and Mark et al concur that resource constraints are a common challenge for military emergency care providers. ${ }^{329,330}$

It remains the sole responsibility of the deploying countries to equip their soldiers with the necessary tools, resources and logistics. ${ }^{331}$ However, it is the host country's responsibility to ensure that peacekeepers have freedom of movement within their borders, which is essential for deploying countries to ship resources and logistics to their deployed soldiers. ${ }^{332}$ Financial constraints and budget cuts in the home country may be a contributing factor to the lack of resources that is experienced by emergency care providers during external deployment. Conversely, host countries offering little or no political support to the mission present another major contributing factor to the lack of resources. ${ }^{333}$ These factors result in management on the ground having to resort to deception. For example, as reported by participants, during inspections equipment is borrowed from other bases in order to trick the inspectors into thinking that the emergency care providers are fully equipped, combat ready and UN compliant. This deception has the negative effect of delaying the supply of equipment to mission areas because senior management and policymakers believe the soldiers on the ground to be fully equipped. Consequently, deployed emergency care providers' skills and capabilities are underutilised, and this contributes to the ongoing practice of deploying poorly resourced emergency care providers to the field. In addition, the participants reported that the equipment that is available is often outdated and in poor condition, which further impedes their ability to perform their duties effectively and efficiently.

The participants described a lack of political will by a host country during deployment which delayed the delivery of resources and supplies to the peace support force. They reported that, during their peace support operation, sustenance flights were often not given landing rights by the host country and this delayed much needed 
resources and supplies. Dandekar and Gow assert that a lack of political will presents an obstacle to a peace support force achieving its mandate during a peace support operation. $^{334}$

They also reported that the host country denied airspace to all flights during Ramadan. A study by Sigri and Basar noted that, during external deployment, deployed military personnel experience hardships owing to a lack of knowledge of foreign cultures, including religion. ${ }^{335}$ Without proper planning and the placement of alternative measures this may result in delays in casualty evacuation to definitive care, therefore strategically disadvantaging military commanders as they may have lesser combat ready troops in their disposal. It may therefore be irrefutable to state that the overall success of a peace support operation depends not only on diplomacy and political momentum but also being well equipped and most importantly planning, improvisation and being able to adapt. ${ }^{336,337}$

Moreover, participants recounted that during external deployment some military emergency care providers felt that, in view of the lack of resources, their only alternative if they were to perform their duties was to purchase lifesaving medication from local pharmacies using their own money. Then they would at least be able to provide much-needed emergency care to the ill and injured. This signifies not only the will and determination of military emergency care providers to provide emergency care to the ill and injured despite their circumstances but also the degree of resource deficiency that currently exists for deployed emergency care providers.

Participants also reported that their initiatives to equip themselves consequently led to exposure to financial exploitation by the local population. This may, however, be the result of economic stagnation, unemployment and poverty. ${ }^{338}$ Moreover, their initiatives are not without strategic consequences. It is important to note that peace support operations are characterised by ever evolving unconventional guerrilla tactics that are not only limited to sniper fire, improvised explosive devices, landmines, but include booby-traps to achieve military objectives and increase peace keeper and civilian casualties. ${ }^{339}$ Therefore the initiative taken by emergency care providers to equip themselves may also make them vulnerable to enemy intelligence operatives by exposing their weakness of resource deficiency. Their vulnerability may lead to a high risk of exposure to unconventional enemy attacks especially as a result of the boobytrapping of purchased items not only by improvised explosives but also weaponised chemical and biological agents. ${ }^{340}$ Moreover their vulnerability may result in civilian casualties as enemy forces may perceive civilian merchants as aids and informants to the peacekeepers.

It is also important to note that in a conflict that results in a collapsed healthcare system, local regulatory bodies that enforce policies have also collapsed. ${ }^{341}$ Therefore there may be no pharmaceutical regulations, which may result in improper storage and handling of medicines. This may result in adverse drug reactions and decreased efficacy during use, which may prolong treatment times, decrease survival to discharge rates, increase the need for referrals to tertiary facilities and therefore have 
a negative impact on emergency care and survival rates, which ultimately results in lesser combat ready soldiers fit for active duty. ${ }^{342}$ Purchasing medications from the black market may also encourage free enterprise in an economically unstable and lawless environment by increasing demand, which promotes profitability from the sale of counterfeit goods and exposes patients receiving emergency care provided by these emergency care providers to counterfeit medicines which are harmful to their health. Moreover, it will also increase the workload and overall threat to peacekeepers as it may encourage the formation of organised crime syndicates specializing in the distribution of medications. These syndicates may utilise their proceeds to increase the funding available to militias to purchase weapons of war, improve their technological capabilities, advance their campaigns, sustain their logistics and supplies and therefore advance their cause even further. This may therefore have a negative impact on the overall success of the peace support operation. Additionally, it may pose a further challenge to humanitarian relief organisations, military personnel and military emergency care providers by increasing the occurrence of ambushes by these militias who use violence to seize the humanitarian relief from the convoys transporting the resources required during the humanitarian assistance operations. ${ }^{343}$

There is also an indication that there is a lack of understanding of the roles and capabilities of deployed emergency care providers by other deployed healthcare professionals. A participant reported that available emergency medications are at times reserved for health care workers who are stationed in static health installations such as sickbays at the base. This results in emergency care providers, who are at the forefront and usually with peacekeepers at the time of injury, being underutilised as they do not have the necessary tools to care for the injured at the time of injury. This may therefore adversely affect morbidity and mortality rates of the injured during ambushes and enemy contact.

A further resource deficit reported by emergency care providers was that of transportation. Participants described how they sometimes had to use combat personnel carriers for casualty transportation. These findings are similar to those of Chapman et al who also found that military emergency care providers use unique casualty transportation during external deployment. ${ }^{344}$ Emergency care providers also sometimes have to use combat vehicles which are not marked with a Red Cross or a Red Crescent owing to the shortage of ambulances which, according to them, is the result of poor maintenance. This is a serious issue because these emergency care providers may not be protected when using an unmarked vehicle as it is not possible to identify them as medical personnel. ${ }^{345}$ Moreover, the use of armoured personnel carriers such as mambas and caspirs, which are equipped with heavy weaponry, may result in military emergency care providers losing protection under the Geneva Convention. According to the International Committee of the Red Cross protocols supplementing the Geneva Conventions resolutions of 12 August 1949, the use of such vehicles is in violation of the responsibilities of medical personnel, which stipulate that these personnel may only carry light weapons for self-defence. ${ }^{346}$ The participants also reported that these armoured personnel carriers are not designed for managing and evacuating casualties and thus do not provide a favourable environment for patient care, nor do they comply with Geneva Convention requirements in respect of casualty evacuation. 


\section{Conclusion}

It is important that the SANDF develop cost-effective and sustainable strategies and means to equip deployed emergency care providers. The SANDF should also raise awareness amongst commanders and other healthcare providers on the importance of emergency care within peace support operations. This will improve the quality of care provided to the ill and injured during external deployment and will also ensure that more South African peacekeepers return home to their families alive and well after heading the call to build a better African continent. Moreover, it will provide a strategic advantage to their role in peacekeeping operations in Africa and assist local populations in these war-torn African countries to receive quality prehospital EMC, thereby increasing the quality of life and thus support nation building.

\section{Limitations}

Access to military emergency care providers was challenging owing to their military commitments. This paper was based on research conducted on a military population based in Gauteng only and thus excluded those in other provinces. Accordingly, the paper discusses only the descriptions of the lived experiences of Gauteng-based emergency care providers of the resource constraints faced during external deployment for the purposes of land-based peace support operations.

\section{Acknowledgements}

The authors would like to thank the participants and the South African Military Health Service for their support and their contributions to this study.

\section{Disclaimer}

The content is solely the responsibility of the authors and does not necessarily represent the official views of the South African National Defence Force, the South African Military Health Service, Durban University of Technology or the University of Pretoria.

\section{Endnotes}

${ }^{306}$ Kime, C. M. 1996. Peacekeeping operations: Maintaining "consent" at the tactical level. Marine Corps Gazette, 80 (2): 48-49.

${ }^{307}$ Ibid.

${ }^{308}$ Buhaug, H. and Urdal, H. 2013. An urbanization bomb? Population growth and social disorder in cities. Global Environmental Change, 23 (1): 1-10.

${ }^{309}$ Bialke, J. P. 2001. United Nations peace operations: Applicable norms and the application of the law of armed conflict. The Air Force Law Review, 50: 1-63.

${ }^{310}$ Johnson, R. J. 2015. Post-cold war United Nations peacekeeping operations: a review of the case for a hybrid level $2+$ medical treatment facility. Disaster and Military Medicine, 1. 
${ }^{311}$ McNally, J. Ligthelm, T., and Martin, S. 2012. Military Health Support Doctrine: Landward Conventional Operations. Simon's Town SA Naval Publication Unit.

${ }^{312}$ Shilcutt, T. M. 2003. First link in the life-chain: Infantry combat medics in Europe, 1944-1945. 3080497, Texas Christian University.

${ }^{313}$ Naidoo, D. 2009.The Fourth Dimension: the untold stories of military health in South Africa. South Africa: South African Military Health Service, Shereno Printers.

${ }^{314}$ McNally, Ligthelm, and Martin. op. cit.

${ }^{315}$ Gilbert, L. 2014. A retrospective examination of post-Cold War Conflicts in Africa: Ended, Abated and Prolonged Comflicts. International Journal of Humanities and Social Science, 4(9): 151-162.

${ }^{316}$ Nibishaka, E. 2011. South Africa's Peacekeeping role in Africa: Motives and challenges of peacekeeping. International Politics, 02.

${ }^{317}$ Johnson, R. 2015. Post- cold war United Nations peacekeeping operations: a review of the case for a hybrid level 2+ medical treatment facility. Disaster and Military Medicine, 1(15): 1-8.

${ }^{318}$ South Africa. Department of Defence. 2014. Annual Report FY 2013/2014. Pretoria: Formeset Print.

${ }^{319}$ Buhaug, and Urdal. op. cit.

${ }^{320}$ Chapman, P. L., Cabrera, L. D., Varela-Mayer, C., Baker, M. M., Elnitsky, C., Figley, C., Thurman, R. M., Lin, C.-D. and Mayer, L. P. 2012. Training, deployment preparation, and combat experiences of deployed health care personnel: key findings from deployed US Army combat medics assigned to line units. Military medicine, 177 (3): 270-277.

${ }^{321}$ Creswell, J. 2013. Qualitative enquiry and research design. 3rd ed. Los Angeles, CA: Sage Publications.

${ }^{322}$ Polit, D.F. and Beck, C.T. 2012. Nursing research: Generating and assessing evidence for nursing practice. 9th edition. London: Wolters.

${ }^{323}$ Oliver, P. 2006. Purposive sampling. The Sage dictionary of social research methods. England: Sage Publications.

${ }^{324}$ Morgan, D. and Given, L. 2008. Sampling. The Sage encyclopedia of qualitative research methods. England: Sage Publications.

${ }^{325}$ Braun, V. and Clarke, V. 2006. Using thematic analysis in psychology. Qualitative Research in Psychology, 3 (2): 77-101.

${ }^{326}$ Creswell. op.cit.

${ }^{327}$ Lewins, A. and Silver, C. 2007. Using software in qualitative research: A step by step guide. Great Britain: Sage Publications.

${ }^{328}$ Sigri, Ü. and Basar, U. 2015. Peacekeeping operations management: An analysis of challenges, changes in command action and training needs. Journal of Defense Resources Management, 6 (2): 13-20. 
${ }^{329}$ Chapman, Cabrera, Varela-Mayer, Baker, Elnitsky, Figley, Thurman, Lin and Mayer. op.cit.

${ }^{330}$ Mark, D.,Connelly, L., Hardy, M., Robison, J., Jones, C. and Streett, T. 2009. Exploring deployment experiences of army medical department personnel. Military Medicine, 174 (6): 631-636.

${ }^{331}$ Bialke. op.cit.

${ }^{332}$ Ibid.

${ }^{333}$ Dandeker, C. and Gow, J. 1997. The future of peace support operations: Strategic peacekeeping and success. Armed Forces and Society, 23 (3): 327-347

${ }^{334}$ Ibid.

${ }^{335}$ Sigri and Basar. op. cit.

${ }^{336}$ Dandeker and Gow. op. cit.

${ }^{337}$ Cullen. op. cit.

${ }^{338}$ Buhaug and Urdal. op.cit.

${ }^{339}$ Schoenfeld, A. J., Lehman Jr, R. A. and Hsu, J. R. 2012. Evaluation and management of combat-related spinal injuries: a review based on recent experiences. The Spine Journal, 12 (9): 817-823.

${ }^{340}$ Erlanger, S. 2009. A Gaza war full of traps and trickery. The New York Times, 11.

${ }^{341}$ Dandeker and Gow. op. cit.

${ }^{342}$ Akici, A., Aydin, V. and Kiroglu, A. 2018. Assessment of the association between drug disposal practices and drug use and storage behaviors. Saudi Pharmaceutical Journal, 26 (1): 7-13.

${ }^{343}$ Langston, V., Gould, M. and Greenberg, N. 2007. Culture: what is its effect on stress in the military? Military Medicine, 172 (9): 931-935.

${ }^{344}$ Chapman, Cabrera, Varela-Mayer, Baker, Elnitsky, Figley, Thurman, Lin and Mayer. op.cit.

${ }^{345}$ Perrin, P. 1996. War and public health: A handbook. Geneva: International Committee of the Red Cross.

${ }^{346}$ International Committee of the Red Cross. 1977. Protocols additional to the Geneva Conventions of 12 August 1949: resolutions of the Diplomatic Conference: extracts from the final act of the Diplomatic Conference. Geneva: International Committee of the Red Cross. 